\title{
Certain types of linear operators on probabilistic Hilbert space
}

\author{
Rana Aziz Yousif Al-Muttalibi*, Radhi Ibrahim M. Ali \\ Department of Mathematics, College of Science for Women, University of Baghdad \\ *Corresponding author E-mail: rana.yousif1982@gmail.com
}

Copyright $\odot 2015$ Rana Aziz Yousif Al-Muttalibi, Radhi Ibrahim M. Ali. This is an open access article distributed under the Creative Commons Attribution License, which permits unrestricted use, distribution, and reproduction in any medium, provided the original work is properly cited.

\begin{abstract}
The purpose of this paper is to introduce some definitions, properties and basic results that show the relation between $F$ bounded of linear operator in probabilistic Hilbert space and bounded operator in norm. In the paper, we prove that the adjoint operator in probabilistic Hilbert space is bounded. The notion of the continuous operators in probabilistic Hilbert space and some basic results are given. In addition, we note that every operator in probabilistic real Hilbert space is a self-adjoint Operator.
\end{abstract}

Keywords: Bounded Operators; Linear Operators; Mathematical Expectation; Probabilistic Hilbert Space; Self-Adjoint Operator.

\section{Introduction}

K. Menger in [10], introduced the notion of probabilistic metric space and since then the theory of probabilistic spaces has been developed in many directions [2], [3]. The notion of probabilistic inner product spaces can be considered as the generalization of the inner product spaces. C. Sklar and B. Schweizer [12] introduced the definition of these spaces in 1983. Similarly, in 1994 S.S., Change [4] introduced the definition of probabilistic inner product space (PIP-space). Then, Yongfu Su in 2001 modified Change's definition in [13]. In 2007, we had also Yongfu su, X.Wang and J. GAO [14] introducing the definition of probabilistic Hilbert space. In 2014 Radhi I. M. Ali and Dalia S. Ali defined the adjoint operator on probabilistic Hilbert space [1]. Guillen. B; Lallena J.; and Sempi. C in [8] had studied the boundedness of linear operators in probabilistic Normed spaces. Their contribution has been quite useful for us in studying the boundedness of linear operator in probabilistic Hilbert space. In [16], the relation between fuzzy continuity and fuzzy boundedness is studied. The definition and relationships between certain types of linear operators in the classical Hilbert space have materialized in some books by some authors [9], [11]. Self-adjoint Operator has several applications in the field of quantum mechanics and in the field of physical observables such as position, momentum, angular momentum and spin [5], [6].

\section{Basic definition}

Definition 2.1 [7] (Distribution function)

A distribution function (d.f.) is a function $\mathrm{F}$ defined on the extended real line $\overline{\mathrm{R}}:[-\infty,+\infty]$ that is non-decreasing and left-continuous, such as that $\mathrm{F}(-\infty)=0$ and $\mathrm{F}(+\infty)=1$. The set of all d.f. will be denoted by $\Delta$; the subset of $\Delta$ formed by the proper d.f.s, i.e. by those d.f.s $\mathrm{F}$ for which.

$\lim _{t \rightarrow-\infty} F(t)=0$ and $\lim _{t \rightarrow+\infty} F(t)=1$

Will be denoted by $\mathrm{D}$.

A special element of $\Delta$ is the function which is defined by 
$H(t)=\left\{\begin{array}{l}0, t \leq 0 \\ 1, t>0\end{array}\right.$

Definition 2.2 [13] (modified probabilistic inner product space) Let $\mathrm{E}$ be a real linear space and let $\mathrm{F}: \mathrm{E} \times \mathrm{E} \rightarrow$ $\mathrm{D}$ be a function, be a function then the modified probabilistic inner product space is the triple $(\mathrm{E}, \mathrm{F}, *)$ where $\mathrm{F}$ is assumed to satisfy the following conditions:

(MPIP-1)

$\mathrm{F}_{\mathrm{x}, \mathrm{X}}(0)=0$,

(MPIP-2)

$\mathrm{F}_{\mathrm{x}, \mathrm{y}}=\mathrm{F}_{\mathrm{y}, \mathrm{x}}$

(MPIP-3)

$F_{x, x}(t)=H(t) \leftrightarrow x=0$,

(MPIP-4)

$F_{\lambda x, y}(t)=\left\{\begin{array}{c}F_{x, y}\left(\frac{t}{\lambda}\right), \lambda>0 \\ H(t), \lambda=0 \\ 1-F_{x, y}(t / \lambda+), \lambda<0\end{array}\right.$

Where $\lambda$ is real number, $F_{x, y}(t / \lambda+)$ is the right hand limit of $F_{x, y}$ at $\frac{t}{\lambda}$.

(MPIP-5)

If $\mathrm{x}$ and $\mathrm{y}$ are linearly independent then

$\mathrm{F}_{\mathrm{x}+\mathrm{y}, \mathrm{z}}(\mathrm{t})=\left(\mathrm{F}_{\mathrm{x}, \mathrm{z}} * \mathrm{~F}_{\mathrm{y}, \mathrm{z}}\right)(\mathrm{t})$

Where

$\left(F_{x, z} * F_{y, z}\right)(t)=\int_{-\infty}^{\infty} F_{x, z}(t-u) d F_{z, y}(u)$

Note that if $\mathrm{x}$ and $\mathrm{y}$ are linearly dependent then

$x+y=x+\alpha x=(1+\alpha) x=\lambda x$ Where $\lambda=1+\alpha$

$F_{x+y, z}(t)=F_{\lambda x, z}(t)=\left\{\begin{array}{c}F_{x, z}\left(\frac{t}{\lambda}\right), \lambda>0 \\ H(t), \lambda=0 \\ 1-F_{x, z}(t / \lambda+), \lambda<0\end{array}\right.$

Which is (MPIP-4).

Then $(E, F, *)$ is the modified probabilistic inner product space.

Remark 2.3 [7] For the definition of probabilistic inner product space, we must depend on distribution functions that belong to $\Delta$ rather than $\Delta^{+}$because this notion should include that of classical inner product space since the inner product space takes negative values; therefore, the set $\Delta$ provides this part.

For all $\mathrm{F}$ in $\Delta$, define the distribution function $\overline{\mathrm{F}}$ in $\Delta$, for all $\mathrm{t} \in \mathrm{R}$, as follows

$\bar{F}(t)=\left\{\begin{array}{c}F(t), \text { if } t \in R, \text { but } F(t) \text { is symmetric } \\ \iota^{-}(1-F(t)), \text { if } t \in R \backslash R^{+}, \text {but } F(t) \text { not symmetric. }\end{array}\right.$ 
Where $\mathrm{t}^{-}(1-\mathrm{F}(\mathrm{t}))$ represents the left limit at $\mathrm{t}$.

Definition 2.4 [14] Let (E, F,*) be a modified PIP-space, If

$\int_{-\infty}^{\infty} \operatorname{tdF}_{\mathrm{x}, \mathrm{y}}(\mathrm{t})$

Is convergent for all $\mathrm{x}, \mathrm{y} \in \mathrm{E}$. Then $(\mathrm{E}, \mathrm{F}, *)$ is called a modified PIP-space with mathematical expectation.

Theorem 2.5: [14] Let $(E, F, *)$ be a modified probabilistic inner product space with mathematical expectation. Let

$<\mathrm{x}, \mathrm{y}>=\int_{-\infty}^{\infty} \mathrm{tdF}_{\mathrm{x}, \mathrm{y}}(\mathrm{t}), \forall \mathrm{x}, \mathrm{y} \in \mathrm{E}$

Then $\quad(\mathrm{E},<>)$ is inner product space, so that $(\mathrm{E},\|\|)$ is inner product space so that $(\mathrm{E},\langle>$ ) is inner product space, so that $(\mathrm{E},\|\|)$, is a normed space where $\|\mathrm{x}\|=\sqrt{\langle\mathrm{x}, \mathrm{x}\rangle} \forall \mathrm{x} \in \mathrm{E}$.

Definition 2.6 [15] Let (E, F,*) be a modified probabilistic inner product space, then:

1) A sequence $\left\{x_{n}\right\}$ in $E$ is said to be $\tau$-convergent to $x \in E$ if $\forall \epsilon>0$ and $\forall \lambda>0 \exists n_{0}(\epsilon, \lambda)$ such that

$$
\mathrm{F}_{\mathrm{x}_{\mathrm{n}}-\mathrm{xx} \mathrm{n}_{\mathrm{n}}-\mathrm{x}}(\epsilon)>1-\lambda \forall \mathrm{n}>\mathrm{n}_{0}(\epsilon, \lambda) .
$$

2) A sequence $\left\{x_{n}\right\}$ in $E$ is called $\tau$-Cauchy convergent if that

$$
\mathrm{F}_{\mathrm{x}_{\mathrm{n}}-\mathrm{x}_{\mathrm{m}} \mathrm{x}_{\mathrm{n}}-\mathrm{x}_{\mathrm{m}}}(\epsilon)>1-\lambda \forall \mathrm{n}, \mathrm{m}>\mathrm{n}_{0}(\epsilon, \lambda) .
$$

3) $(\mathrm{E}, \mathrm{F}, *)$ is and such as that. to be $\tau$-complete if each $\tau$-Cauchy sequence in $\mathrm{E}$ is $\tau$-convergent in $\mathrm{E}$. Definition 2.7 [14] Let $(\mathrm{E}, \mathrm{F}, *)$ be a modified probabilistic inner product space then a linear functional $\mathrm{T}$ defined on $\mathrm{E}$ is said to be continuous if for all sequence $\left\{x_{n}\right\} \subseteq E$ that $\tau$-converges to $x \in E$ then $T\left(x_{n}\right) \rightarrow T(x)$.

Theorem 2.8: [14] (Riesz representation theorem) Let $(E, F, *)$ be a modified probabilistic Hilbert space, for any linear continuous functional $f(x)$ there exists a unique $y \in E$ such that:

$\mathrm{f}(\mathrm{x})=\int_{-\infty}^{\infty} \mathrm{tdF}_{\mathrm{x}, \mathrm{y}}(\mathrm{t}) \forall \mathrm{x} \in \mathrm{E}$

Theorem 2.9: [1] (adjoint operator on probabilistic Hilbert space) Let $(E, F, *)$ be a modified probabilistic Hilbert space, Let $T$ be a continuous linear operator on $(E, F, *)$ then there exists a unique $T^{*} \in(E, F, *)$ linear operator such that

$<\mathrm{Tx}, \mathrm{y}>=<\mathrm{x}, \mathrm{T}^{*} \mathrm{y}>\forall \mathrm{x}, \mathrm{y} \in \mathrm{E}$

Theorem 2.10: [1] (properties of the adjoint operator) Let $(E, F, *)$ be a modified probabilistic Hilbert space, let $T^{*}$ be the adjoint operator of $T$, then $T^{*}$ has the following properties:

1) $\quad\left(\mathrm{T}^{*}\right)^{*}=\mathrm{T}$

2) $(\alpha \mathrm{T})^{*}=\alpha \mathrm{T}^{*}$

3) $\quad\left(\mathrm{T}_{1}+\mathrm{T}_{2}\right)^{*}=\mathrm{T}_{1}^{*}+\mathrm{T}_{2}^{*}$

4) $\quad\left(\mathrm{T}_{1} \mathrm{~T}_{2}\right)^{*}=\mathrm{T}_{2}^{*} \mathrm{~T}_{1}^{*}$

Definition 2.11 [9] Let $\mathrm{H}$ be Hilbert space and let $\mathrm{T}: \mathrm{H} \rightarrow \mathrm{H}$ be bounded linear operator then $\mathrm{T}$ is:

1) Self-adjoint operator if $\mathrm{T}=\mathrm{T}^{*}$.

2) Unitary operator if $\mathrm{TT}^{*}=\mathrm{I}=\mathrm{T}^{*} \mathrm{~T}$.

3) Isometric operator if $\mathrm{T}^{*} \mathrm{~T}=\mathrm{I}$

Definition 2.12 [11] (bounded in norm) Let $\mathrm{T}$ be a linear operator defined on normed space $\mathrm{E}$, then $\mathrm{T}$ is said to be bounded in norm if there exists a constant $\mathrm{M}>0$ such that

\| Tx $\|\leq M\| x \|$ for all $\mathrm{x} \in \mathrm{E}$ 


\section{Main results}

This part, defines the self-adjoint operator in probabilistic Hilbert space; the relationship between this operator and the other operators is also shown. We have been able to find the relation between the F-bounded operator and the bounded operator in norm and are going to use it to prove that the adjoint operator is bounded. The notion of F-continuous operator and some basic results are given.

\section{1. (Self-adjoint operator in probabilistic Hilbert space)}

Definition 3.1.1 let $(\mathrm{E}, \mathrm{F}, *)$ be a modified probabilistic Hilbert space with mathematical expectation and let T: (E, F,* )$\rightarrow(\mathrm{E}, \mathrm{F} *)$ be a linear operator then $\mathrm{T}$ is:

1) Self-adjoint operator if $\mathrm{T}=\mathrm{T}^{*}$.

2) Unitary operator if $\mathrm{TT}^{*}=\mathrm{I}=\mathrm{T}^{*} \mathrm{~T}$.

3) Isometric operator if $\mathrm{T}^{*} \mathrm{~T}=\mathrm{I}$.

The following theorem shows that every operator in probabilistic Hilbert space is a Self-Adjoint operator.

Theorem 3.1.2: Let $(E, F, *)$ be a modified probabilistic Hilbert space with mathematical expectation and let $T:(E, F, *$ )$\rightarrow(E, F *)$ be a linear operator then

1) T Is Self-adjoint operator.

2) $\|\mathrm{Tx}\|=\left\|\mathrm{T}^{*} \mathrm{x}\right\|$ for all $\mathrm{x} \in \mathrm{E}$

Proof: (1) Since E is real linear space, and

$<\mathrm{Tx}, \mathrm{x}>=\int_{-\infty}^{\infty} \mathrm{tdF}_{\mathrm{Tx}, \mathrm{x}}(\mathrm{t})<\infty$

Then

$<\mathrm{Tx}, \mathrm{x}>$ is real for all $\mathrm{x} \in \mathrm{E}$.

$<\mathrm{Tx}, \mathrm{x}>=\overline{<\mathrm{Tx}, \mathrm{x}>}$

$<\mathrm{Tx}, \mathrm{x}>-\overline{<\mathrm{Tx}, \mathrm{x}>}=0$

$\int_{-\infty}^{\infty} \mathrm{tdF}_{\mathrm{Tx}, \mathrm{x}}(\mathrm{t})-\int_{-\infty}^{\infty} \mathrm{tdF}_{\mathrm{x}, \mathrm{Tx}}(\mathrm{t})=0$

$\int_{-\infty}^{\infty} \mathrm{tdF}_{\mathrm{Tx}, \mathrm{x}}(\mathrm{t})-\int_{-\infty}^{\infty} \mathrm{tdF}_{\mathrm{T}^{*} \mathrm{x}, \mathrm{x}}(\mathrm{t})=0$

$\int_{-\infty}^{\infty} \mathrm{tdF}_{\mathrm{Tx}-\mathrm{T}^{*} \mathrm{x}, \mathrm{x}}(\mathrm{t})=0$

Since $\mathrm{T}, \mathrm{T}^{*}$ are linear

$\int_{-\infty}^{\infty} \operatorname{tdF}_{\left(\mathrm{T}-\mathrm{T}^{*}\right) \mathrm{x}, \mathrm{x}}(\mathrm{t})=0$

$\left(\mathrm{T}-\mathrm{T}^{*}\right) \mathrm{x}=0 \forall \mathrm{x} \in \mathrm{E}$

$\mathrm{T}-\mathrm{T}^{*}=0$

$\mathrm{T}=\mathrm{T}^{*}$

Then $\mathrm{T}$ is self-adjoint operator.

2. Since $\mathrm{T}$ is Self-Adjoint then $\mathrm{T}^{*} \mathrm{~T}=\mathrm{TT}^{*}$

$<\mathrm{T}^{*} \mathrm{Tx}, \mathrm{x}>=<\mathrm{TT}^{*} \mathrm{x}, \mathrm{x}>\forall \mathrm{x} \in \mathrm{E}$

$\int_{-\infty}^{\infty} \operatorname{tdF}_{\mathrm{T}^{*} T \mathrm{~T}, \mathrm{x}}(\mathrm{t})=\int_{-\infty}^{\infty} \mathrm{tdF}_{\mathrm{TT}^{*} \mathrm{x}, \mathrm{X}}(\mathrm{t})$

$\int_{-\infty}^{\infty} \mathrm{tdF}_{\mathrm{Tx}, \mathrm{Tx}}(\mathrm{t})=\int_{-\infty}^{\infty} \mathrm{tdF}_{\mathrm{T}^{*} \mathrm{x}, \mathrm{T}^{*} \mathrm{x}}(\mathrm{t})$ 
$<\mathrm{Tx}, \mathrm{Tx}>=<\mathrm{T}^{*} \mathrm{x}, \mathrm{T}^{*} \mathrm{x}>$

$\|\mathrm{Tx}\|=\left\|\mathrm{T}^{*} \mathrm{x}\right\|$

Remark 3.1.3 let $(\mathrm{E}, \mathrm{F}, *)$ be a modified probabilistic Hilbert space with mathematical expectation and let $\mathrm{T}:(\mathrm{E}, \mathrm{F}, *) \rightarrow$ $(\mathrm{E}, \mathrm{F} *)$ be a linear operator then

1) If $\mathrm{T}$ is unitary, then $\mathrm{T}$ and $\mathrm{T}^{*}$ are isometric operators.

2) $T$ is unitary if and only if $T^{*}$ is unitary.

Proposition 3.1.4 Let $(\mathrm{E}, \mathrm{F}, *)$ be a modified probabilistic Hilbert space with mathematical expectation, and let T: (E, F,* )$\rightarrow(\mathrm{E}, \mathrm{F} *)$ be a linear operator, If $\mathrm{T}$ is self-adjoint operator that satisfies $\mathrm{T}^{2}=\mathrm{I}$ then $\mathrm{T}$ is unitary and isometric.

Proof: If $\mathrm{T}$ is self-adjoint operator then $\mathrm{T}=\mathrm{T}^{*}$, multiplying this equation by $\mathrm{T}$ from both sides, we get $\mathrm{TT}=\mathrm{T} * \mathrm{~T}$ but $\mathrm{T}^{2}=\mathrm{I}$ then $\mathrm{TT}^{*}=\mathrm{I}=\mathrm{T}^{*} \mathrm{~T}$, hence $\mathrm{T}$ is unitary.

Also, since $\mathrm{T}^{2}=\mathrm{I}$ and $\mathrm{T}$ is self-adjoint Operator then $\mathrm{T}^{*} \mathrm{~T}=\mathrm{I}$, hence $\mathrm{T}$ is isometric.

\subsection{The boundedness of linear operators in probabilistic Hilbert space.}

The idea of the following definition is similar to the idea of B-bounded, which is introduced in [8]; we have used the distribution function in probabilistic Hilbert space $\left(\mathrm{F}_{\mathrm{x}, \mathrm{y}}(\mathrm{t})\right)$ instead of the distribution function $\left(\mathrm{v}_{\mathrm{p}}(\mathrm{x})\right)$, which is defined on probabilistic normed space.

Definition 3.2.1 ( F-bounded operator) Let $(\mathrm{E}, \mathrm{F}, *)$ be a modified probabilistic Hilbert space with mathematical expectation, let $\mathrm{T}$ be a linear operator defined on $(\mathrm{E}, \mathrm{F}, *)$ then $\mathrm{T}$ is said to be $\mathrm{F}$-bounded Operator if there exists a constant $\mathrm{K}>0$, such that

$\mathrm{F}_{\mathrm{Tx}, \mathrm{Tx}}(\mathrm{t}) \geq \mathrm{F}_{\mathrm{X}, \mathrm{x}}\left(\frac{\mathrm{t}}{\mathrm{K}}\right)$ for all $\mathrm{x} \in \mathrm{E}, \mathrm{t} \in \mathrm{R}$

Lemma 3.2.2: Let $(E, F, *)$ be a modified probabilistic Hilbert space with mathematical expectation and if $F$ and $G$ are two functions in $\Delta$ such that $\mathrm{F}(\mathrm{t}) \leq \mathrm{G}(\mathrm{t})$, then

$\int_{-\infty}^{\infty} \operatorname{tdF}(\mathrm{t}) \geq \int_{-\infty}^{\infty} \mathrm{tdG}(\mathrm{t})$

Proof: Since $F(t) \leq G(t)$, then by using the definition (2.1) and integration by parts, we get

$\int_{-\infty}^{\infty} \mathrm{tdF}(\mathrm{t}) \geq \int_{-\infty}^{\infty} \mathrm{tdG}(\mathrm{t})$

Theorem 3.2.3: Let $(E, F, *)$ be a modified Probabilistic Hilbert Space with mathematical expectation, let $T$ be a linear operator defined on $(E, F, *)$, then $T$ is $F$-bounded Operator if and only if $T$ is bounded in norm on $E$.

Proof: Assume that T is F -bounded then

$\mathrm{F}_{\mathrm{Tx}, \mathrm{Tx}}(\mathrm{t}) \geq \mathrm{F}_{\mathrm{X}, \mathrm{x}}\left(\frac{\mathrm{t}}{\mathrm{K}}\right)$ for all $\mathrm{x} \in \mathrm{E}$, where $\mathrm{K}>0$

$\|\mathrm{Tx}\|^{2}=<\mathrm{Tx}, \mathrm{Tx}>=\int_{-\infty}^{\infty} \mathrm{td} \mathrm{F}_{\mathrm{Tx}, \mathrm{Tx}}(\mathrm{t})$

By lemma (3.2.2), we have

$$
\begin{aligned}
& \int_{-\infty}^{\infty} \operatorname{td~} F_{T x, T x}(t) \leq \int_{-\infty}^{\infty} \operatorname{td~F}_{x, x}\left(\frac{t}{K}\right) \\
& =K \int_{-\infty}^{\infty} \operatorname{td~F}_{x, x}(t) \\
& =K<x, x>
\end{aligned}
$$


$=\mathrm{K}\|\mathrm{x}\|^{2}$

$\|\mathrm{Tx}\|^{2} \leq \mathrm{K}\|\mathrm{x}\|^{2}$ for all $\mathrm{x} \in \mathrm{E}$, where $\mathrm{K}>0$ is a constant,

$\|\mathrm{Tx}\| \leq \sqrt{\mathrm{K}}\|\mathrm{x}\|$

Then $\mathrm{T}$ is bounded in norm on $\mathrm{E}$.

Assume that $\mathrm{T}$ is bounded in norm, then there exists a constant $\mathrm{M}>0$ such that

$\|\mathrm{Tx}\| \leq \mathrm{M}\|\mathrm{x}\|$ for all $\mathrm{x} \in \mathrm{E}$

$\|\mathrm{Tx}\|^{2}=<\mathrm{Tx}, \mathrm{Tx}>=\int_{-\infty}^{\infty} \mathrm{td} \mathrm{F}_{\mathrm{Tx}, \mathrm{Tx}}(\mathrm{t})$

$\leq \mathrm{M} \int_{-\infty}^{\infty} \mathrm{td} \mathrm{F}_{\mathrm{x}, \mathrm{X}}(\mathrm{t})$

$=\int_{-\infty}^{\infty} t d F_{x, x}\left(\frac{t}{M}\right)$

By lemma 3.2.2, we have

$F_{T X, T X}(t) \geq F_{X, X}\left(\frac{t}{M}\right)$ for all $x \in E$, where $M>0$

Then $\mathrm{T}$ is F-bounded Operator.

Remark 3.2.4 Let $(\mathrm{E}, \mathrm{F}, *)$ be a modified probabilistic Hilbert space with mathematical expectation, let $\mathrm{T}$ be a linear operator defined on $(\mathrm{E}, \mathrm{F}, *)$, and $\mathrm{T}^{*}$ is the adjoint operator of $\mathrm{T}$ then:

1) If $\mathrm{T}$ is F-bounded operator then $\mathrm{T}^{*}$ is F-bounded operator.

2) If $\mathrm{T}$ is bounded in norm on $\mathrm{E}$ then $\mathrm{T}^{*}$ is bounded in norm on $\mathrm{E}$.

Proof: (1) Assume that T is F-bounded operator then

$F_{T x, T x}(t) \geq F_{x, x}\left(\frac{t}{K}\right)$ for all $x \in E$, where $K>0$

By theorem (3.1.2)

$F_{T^{*} x, T^{*} x}(t) \geq F_{x, x}\left(\frac{t}{K}\right)$

Then $\mathrm{T}^{*}$ is F-bounded operator.

Proof (2) Let $\mathrm{T}$ is bounded in norm on $\mathrm{E}$ then for some $\mathrm{M}>0$, we have

$\|\mathrm{Tx}\| \leq \mathrm{M}\|\mathrm{x}\|$

$\|\mathrm{Tx}\|^{2} \leq \mathrm{M}^{2}\|\mathrm{x}\|^{2}$

$\int_{-\infty}^{\infty} \mathrm{td} \mathrm{F}_{\mathrm{TX}, \mathrm{Tx}}(\mathrm{t}) \leq \int_{-\infty}^{\infty} \mathrm{td} \mathrm{F}_{\mathrm{x}, \mathrm{x}}\left(\frac{\mathrm{t}}{\mathrm{M}^{2}}\right)$

By theorem (3.1.2)

$\int_{-\infty}^{\infty} \mathrm{td} \mathrm{F}_{\mathrm{T}^{*} \mathrm{x}, \mathrm{T}^{*} \mathrm{x}}(\mathrm{t}) \leq \int_{-\infty}^{\infty} \mathrm{td} \mathrm{F}_{\mathrm{x}, \mathrm{x}}\left(\frac{\mathrm{t}}{\mathrm{M}^{2}}\right)$

$\left\|\mathrm{T}^{*} \mathrm{x}\right\|^{2} \leq \mathrm{M}^{2}\|\mathrm{x}\|^{2}$

$\left\|\mathrm{T}^{*} \mathrm{x}\right\| \leq \mathrm{M}\|\mathrm{x}\|$

Then $\mathrm{T}^{*}$ is bounded in norm on $\mathrm{E}$. 


\subsection{The continuity of linear operators in probabilistic Hilbert space.}

Definition 3.3.1 ( $\mathrm{F}$-continuous operator) Let $(\mathrm{E}, \mathrm{F}, *)$ be a modified probabilistic Hilbert space with mathematical expectation and let $\mathrm{T}:(\mathrm{E}, \mathrm{F}, *) \rightarrow(\mathrm{E}, \mathrm{F} *)$ be a linear operator, then $\mathrm{T}$ is said to be $\mathrm{F}$-continuous at $\mathrm{y} \in \mathrm{E}$ if for all $\epsilon>0$ there exists corresponding $\delta>0$ such that for all $\mathrm{x} \in \mathrm{E}$ we have.

$\mathrm{F}_{\mathrm{Tx}-\mathrm{Ty}, \mathrm{Tx}-\mathrm{Ty}}(\epsilon) \geq \mathrm{F}_{\mathrm{x}-\mathrm{y}, \mathrm{x}-\mathrm{y}}(\delta)$

If $\mathrm{T}$ is $\mathrm{F}$-continuous at each point of $\mathrm{E}$ then $\mathrm{T}$ is $\mathrm{F}$-continuous on $(\mathrm{E}, \mathrm{F}, *)$.

Theorem 3.3.2: Let $(E, F, *)$ be a modified probabilistic Hilbert space with mathematical expectation, and let $T:(E, F, *$ )$\rightarrow(E, F *)$ be a linear operator, then if $T$ is $F$-continuous operator on $(E, F, *)$ then $T$ is continuous on $E$.

Proof: Since $\mathrm{T}$ is $\mathrm{F}$-continuous on $(\mathrm{E}, \mathrm{F}, *)$, then $\mathrm{T}$ is continuous at each point of $\mathrm{E}$. Suppose that $\mathrm{T}$ is $\mathrm{F}$-continuous at $\mathrm{x}_{0} \in \mathrm{E}$, then for all $\epsilon>0$ there exists corresponding $\delta>0$ such that for all $\mathrm{x} \in \mathrm{E}$, we have.

$\mathrm{F}_{\mathrm{Tx}-\mathrm{Tx}_{0}, \mathrm{Tx}-\mathrm{Tx}_{0}}(\epsilon) \geq \mathrm{F}_{\mathrm{x}-\mathrm{x}_{0}, \mathrm{X}-\mathrm{x}_{0}}(\delta)$

Let $\left\{\mathrm{x}_{\mathrm{n}}\right\}$ be a sequence of elements of $\mathrm{E}$ i.e. $\left\{\mathrm{x}_{\mathrm{n}}\right\} \subseteq \mathrm{E}$ that's $\mathrm{x}_{\mathrm{n}}$ is $\tau$ - converges to $\mathrm{x}_{0}$ thus for all $\delta>0, \lambda>0$ there exists $\mathrm{n}_{0}(\delta, \lambda)$ such that

$\mathrm{F}_{\mathrm{x}_{\mathrm{n}}-\mathrm{x}_{0} \mathrm{x}_{\mathrm{n}}-\mathrm{x}_{0}}(\delta)>1-\lambda \forall \mathrm{n}>\mathrm{n}_{0}(\delta, \lambda)$

Then

$\mathrm{F}_{\mathrm{Tx}_{\mathrm{n}}-\mathrm{Tx}_{0}, \mathrm{Tx}_{\mathrm{n}}-\mathrm{Tx}_{0}}(\epsilon) \geq \mathrm{F}_{\mathrm{x}_{\mathrm{n}}-\mathrm{x}_{0} \mathrm{x}_{\mathrm{n}}-\mathrm{x}_{0}}(\delta)>1-\lambda \forall \mathrm{n}>\mathrm{n}_{0}(\delta, \lambda)$

Then $\mathrm{T}$ is continuous on $\mathrm{E}$.

Theorem 3.3.3: Let $(E, F, *)$ be a modified probabilistic Hilbert space with mathematical expectation and let $T:(E, F, *$ )$\rightarrow(E, F *)$ be a linear operator, then $T$ is $F$-bounded operator if and only if $T$ is $F$-continuous operator.

Proof: Assume that $\mathrm{T}$ is -the operator, $\mathrm{t}$-bounded en there exists $\mathrm{K}>0$ such that for all $\mathrm{x} \in \mathrm{E}, \mathrm{t} \in \mathrm{R}$, we have

$$
\mathrm{F}_{\mathrm{Tx}, \mathrm{Tx}}(\mathrm{t}) \geq \mathrm{F}_{\mathrm{X}, \mathrm{X}}\left(\frac{\mathrm{t}}{\mathrm{K}}\right)
$$

For have. $\in>0$, We can choose $\delta=\frac{\epsilon}{\mathrm{K}} \quad$ for $\quad \epsilon=|\mathrm{t}|>0 \quad$ we $\quad$ have

$\mathrm{F}_{\mathrm{Tx}-\mathrm{Ty}, \mathrm{Tx}-\mathrm{Ty}}(\epsilon) \geq \mathrm{F}_{\mathrm{x}-\mathrm{y}, \mathrm{x}-\mathrm{y}}(\delta)$

In case $|t|=0$, we have

$\mathrm{F}_{\mathrm{Tx}-\mathrm{Ty}, \mathrm{Tx}-\mathrm{Ty}}(\epsilon)=0=\mathrm{F}_{\mathrm{x}-\mathrm{y}, \mathrm{x}-\mathrm{y}}(\delta)$

Then $\mathrm{T}$ is F-continuous operator on $(\mathrm{E}, \mathrm{F}, *)$.

Conversely, assume that $\mathrm{T}$ is $\mathrm{F}$-continuous operator on $(\mathrm{E}, \mathrm{F}, *)$, then $\mathrm{T}$ is $\mathrm{F}$-continuous at 0 , thus for all $\epsilon>0$ there exists corresponding $\delta>0$ such that for all $\mathrm{x} \in \mathrm{E}$, we have.

$\mathrm{F}_{\mathrm{Tx}, \mathrm{Tx}}(\epsilon) \geq \mathrm{F}_{\mathrm{X}, \mathrm{x}}(\delta)$

If $x \neq 0$, put $\epsilon=|t|$ and $\delta=\frac{\epsilon}{\mathrm{K}}, \mathrm{t} \in \mathrm{R} \backslash\{0\}$, then

$\mathrm{F}_{\mathrm{TX}, \mathrm{Tx}}(\mathrm{t}) \geq \mathrm{F}_{\mathrm{X}, \mathrm{x}}\left(\frac{\mathrm{t}}{\mathrm{K}}\right)$

In case $t=0$, we have 
$\mathrm{F}_{\mathrm{Tx}, \mathrm{Tx}}(\mathrm{t})=0=\mathrm{F}_{\mathrm{x}, \mathrm{x}}\left(\frac{\mathrm{t}}{\mathrm{K}}\right)$

If $x=0$ and $t \in R \backslash\{0\}$, then

$\mathrm{F}_{\mathrm{TX}, \mathrm{TX}}(\mathrm{t})=\mathrm{H}(\mathrm{t})=\mathrm{F}_{\mathrm{X}, \mathrm{x}}\left(\frac{\mathrm{t}}{\mathrm{K}}\right)$.

In case $t=0$, we have.

$\mathrm{F}_{\mathrm{TX}, T \mathrm{Tx}}(\mathrm{t})=0=\mathrm{F}_{\mathrm{X}, \mathrm{x}}\left(\frac{\mathrm{t}}{\mathrm{K}}\right)$.

Then $\mathrm{T}$ is F-bounded operator.

Theorem 3.3.4: Let $(E, F, *)$ be a modified probabilistic Hilbert space with mathematical expectation and let $T:(E, F, *$ )$\rightarrow(E, F *)$ be a linear operator, then $T$ is $F$-continuous operator on $(E, F, *)$ if and only if $T$ is continuous in norm on E.

Proof: Suppose that $\mathrm{T}$ is $\mathrm{F}$-continuous operator on $(\mathrm{E}, \mathrm{F}, *)$, then by theorem (3.3.2), $\mathrm{T}$ is continuous operator on $(\mathrm{E}, \mathrm{F}, *)$. Thus, for all sequence $\left\{\mathrm{x}_{\mathrm{n}}\right\} \subseteq \mathrm{E}$ that $\tau$-converges to $\mathrm{x} \in \mathrm{E}$ we have $\mathrm{T}\left(\mathrm{x}_{\mathrm{n}}\right) \rightarrow \mathrm{T}(\mathrm{x})$, by theorem (2.4), we get $\mathrm{T}$ is continuous in norm on $\mathrm{E}$.

Conversely, suppose that $\mathrm{T}$ is continuous in norm $\mathrm{E}$, then $\mathrm{T}$ is bounded in norm on $\mathrm{E}$. By theorem (3.2.3), we get $\mathrm{T}$ is $\mathrm{F}$ bounded operator, and, hence, by previous theorem (3.3.3), we have T is F-continuous operator on (E, F,*).

\section{Conclusion}

In this paper, we have defined some new types of linear operators on probabilistic Hilbert space and established the relationship between them. The paper tries to prove the boundedness of the adjoint operator in probabilistic Hilbert space.

\section{Acknowledgements}

First, I am grateful to Allah the most merciful, the most compassionate for enabling me to write this paper. I would like to express my gratitude to my professors at the Department of Mathematics, to my family and, in particular, to my daughter, and, equally, to Dalia Sami, my intimate friend and her family as well.

\section{References}

[1] Al-Saady Ali D. and Mohammed Ali R (2014). Adjoint Operator in Probabilistic Hilbert Space, Mathematical Theory and Modeling, pp: 1017.

[2] Alsina, C.; Schweizer B.; and Sklar, A. (1993). On the Definition of Probabilistic Normed Space. Aequationes Mathematicae, pp: 91-98. http://dx.doi.org/10.1007/BF01834000.

[3] Alsina, C.; Schweizer, B.; Sklar, A.; and Sempi. C. (1997). On the Definition of a Probabilistic Inner Product Space, Rendiconti di Matematica, Serie pp: 115-127.

[4] Chang S., Cho Y., Kang M. S. (1994). Probabilistic Metric Spaces and Nonlinear Operators Theory, Sichuan university press.

[5] El-Naschie, M. (1998). On the Uncertainty of Cantorian Geometry and Two-slit Experiment, Chaos, Solutions and Fractals, pp: 517-529.

[6] El-Naschie, M. (2000). On the Unication of Heterotic Strings, M Theory and 1 Theory, Chaos, Solitons and Fractals, pp: $2397-2408$. http://dx.doi.org/10.1016/S0960-0779(00)00108-9.

[7] Guillen, B., Harikrishnam, P., (2014). Probabilistic Normed Spaces. London, Imperial College Press. http://dx.doi.org/10.1142/p944.

[8] Guillen. B; Lallena J.; and Sempi. C, (1999). A study of Boundedness in Probabilistic Normed Spaces, Journal of mathematical Analysis and application, pp: 183-196. http://dx.doi.org/10.1006/jmaa.1998.6261.

[9] Kreyszig, E. (1989). Introductory Functional Analysis with Applications, Wiley Classics Library Edition.

[10] Menger, K. (1942). Statistical Metrics, Proceedings of the National Academy of Sciences, pp: 535-537. http://dx.doi.org/10.1073/pnas.28.12.535.

[11] Rudin, W. (1991). Functional Analysis, McGraw-Hill Science.

[12] Schweizer, B.; and Sklar, A. (1983). Probabilistic Metric Spaces, New York, Amsterdam, Oxford: North Holland.

[13] Su, Y. (2001). On Definition of Probabilistic Inner Product Spaces, Acta Analysis Functionalis Applicate, pp: $193-196$.

[14] Su, Y.; Wang, X.; and GAO, J. (2007). Riesz Theorem in Probabilistic Inner Product Spaces, International Mathematical Forum, pp: 30733078 .

[15] Su, Y.; Wang, X.; and GAO, J. (2008). Approximation of Fixed Points for Nonlinear Operators in Probabilistic Inner Product Space. International Journal of Applied Mathematics, pp: 1-9.

[16] T. Bag, S.K. Samanta. (2005). Fuzzy bounded linear operators, Fuzzy Sets and Systems pp: 513-547. http://dx.doi.org/10.1016/j.fss.2004.05.004. 